\title{
Maltrato y autoestima de adolescentes Awajún de Chipe Cuzu, región Amazonas, Perú, 2021
}

\section{Mistreatment and self-esteem of Awajún adolescents of Chipe Cuzu, Amazonas region, Peru, 2021}

\author{
Riomer Pasesku Petsayit ${ }^{1}$, Taisia Marlith Landacay Laulate² ${ }^{2}$ Zally Santillán Sánchez ${ }^{3}$
}

\section{RESUMEN}

El objetivo de la presente investigación fue determinar la relación entre el maltrato y autoestima de adolescentes Awajún de Chipe Cuzu, región Amazonas, Perú, 2021. El enfoque fue cuantitativo, con un nivel relacional; de corte transversal y orientación explicativa. La muestra estuvo representada por 20 adolescentes Awajún, matriculados en el semestre académico 2021, a quieres se les aplicó el test de maltrato y el inventario de autoestima Forma Escolar Coopersmith, ambos fueron aplicados de forma presencial, donde los evaluados tuvieron que marcar las respuestas, el primer instrumento con 36 proposiciones que identificó los tipos de maltrato infantil, con 4 formas: maltrato infantil físico, maltrato infantil emocional o psicológico, maltrato infantil de desatención o negligencia y maltrato sexual, mientras que el segundo, con 58 proposiciones a las que se respondió con Sí o No, divido en 4 subescalas: área sí mismo, área social, área hogar y área. Encontrándose que, existe una correlación de $\mathrm{r}=-0.522$ entre maltrato infantil y autoestima, de 20 adolescentes Awajún el $95 \%$ ha padecido de maltrato psicológico (19) y el 85\% (17) poseen una autoestima promedio. Se concluyó que hay una relación significativa al $5 \%$ entre maltrato y autoestima.

Palabras clave: Adolescente, maltrato, autoimagen

\begin{abstract}
The present research whose aimed to find the relationship between mistreatment and self-esteem Awajún adolescents of Chipe Cuzu in Amazonas region, Perú, 2021. A quantitative approach, a relational, cross-sectional and explanatory. Twenty (20) students enrolled in the 2021 semester were analyzed. Were administered the mistreatment test and the original Coopersmith School Form Self-Esteem Inventory were applied, both were applied in person, where the evaluated had to mark the answers, the first instrument with 36 propositions that identified the types of child mistreatment, with 4 forms: physical child abuse, emotional or psychological child abuse, child abuse of neglect or negligence and sexual abuse, while the second, with 58 propositions that were answered with Yes or No, divided into 4 subscales: self area, social area, home area and area. Results indicate a correlation $(\mathrm{r}=0.522)$ between mistreatment and level of self-esteem, as $95 \%$ of subjects have suffered mistreatment and $85 \%$ possess an average self-esteem. Therefore, the level of self-esteem is significantly associated with maltreatment by $5 \%$.
\end{abstract}

Keywords: Adolescent, mistreatment, self-esteem

\footnotetext{
${ }^{1}$ Estudiante de pregrado de la Carrera profesional de Psicología. Facultad Ciencias de la Salud-UNTRM. Email: riomerpasesku@gmail.com ${ }^{2}$ Estudiante de pregrado de la Carrera profesional de Psicología. Facultad Ciencias de la Salud-UNTRM. Email: taislandacay@gmail.com ${ }^{3}$ Estudiante de pregrado la Carrera profesional de Psicología. Facultad Ciencias de la Salud-UNTRM. Email: santillansanchezzally@gmail.com
} 


\section{INTRODUCCIÓN}

El maltrato infantil engloba a la población menor de 18 años y se efectúa en un contexto de relación de confianza, poder o responsabilidad cuando se causa un daño al proceso de crecimiento saludable e íntegro del menor o en la gravedad del caso, al poner en riesgo su vida; así mismo existen múltiples formas en las que se da el maltrato, como físico, psicológico, abuso sexual y desatención(OMS, 2020).

En consecuencia, en algunos lugares se sigue cometiendo, a pesar de tener conocimiento sobre los daños que causa, hay culturas que lo normalizan como una forma necesaria para ejercer disciplina y una buena formación del menor.

Sin embargo, es un mal que no solo afecta a países están empezando a desarrollarse, sino que también afecta a aquellos más desarrollados, de esta manera se convierte en un problema mundial de salud que ha conllevado a la creación de múltiples organismos para contrarrestar el aumento significativo del problema. Sin embargo, a pesar de los esfuerzos para frenar el abuso infantil los datos registrados van cada vez en aumento.

Así mismo, el maltrato trae consigo graves consecuencias no solo a nivel físico o psicológico, sino también a nivel neurobiológico. (AmoresVillalba y Mateos-Mateos, 2017). Así como lo demuestran diversas investigaciones, sus consecuencias se pueden ver expresadas mayormente en el estrés, ansiedad, depresión y baja autoestima.

Por otro lado, la autoestima viene a ser cómo una persona se concibe y valora a ella misma, en esta se ven inmersas pensamientos, actitudes, emociones e ideas que ha aprendido y ha implantado como verdaderas. (Mejía et al, 2011 citado por Arango y Panesso, 2017). Abarca el autoconcepto y autoimagen, donde acepta que es un ser capaz de funcionar como cualquier otro, llamándole a esto autoeficacia y es capaz de tener confianza en que sus deseos, emociones e ideas son las mismas que las de cualquiera y tienen la misma importancia, ésta se va construyendo a lo largo de la vida a partir de la interacción humana (Pérez, 2019). Está presente en distintos niveles y dependiendo de ello va a repercutir de forma positiva o negativa respectivamente, afectando tanto a nivel personal y social del individuo. (Arango y Panesso, 2017)

De acuerdo a los estudios de nivel internacional realizado en 96 países sobre la prevalencia del maltrato infantil, se encontraron que 1000 millones de infantes de ambos sexos, de 2 a 17 años, fueron víctimas del maltrato infantil de tipo físico, emocional y sexual. Otro de los indicadores más preocupantes está relacionado con la falta de responsabilidad de los agentes que pueden llevar a cabo una denuncia, esta realidad se evidencia en un metaanálisis de los datos mundiales, donde se observó que la denuncia llevada a cabo por los mismos niños y niñas por el tema del abuso sexual es 30 veces más alta y 75 veces más alto en abuso físico en comparación de los registros oficiales (OPS, 2016).

En el ámbito nacional en el 2018 el Centro Emergencia Mujer atendió más de 41 mil casos de abuso contra población infantil. Así mismo, en el 2019 de enero a setiembre se reportó más de $40 \mathrm{mil}$ casos de violencia infantil. De los cuales más de 8 mil incidentes corresponden a maltrato sexual, de estos, más de 3 mil casos ocurrieron contra la población infantil femenina (Defensoría del pueblo, 2019). Así mismo, de acuerdo a la encuesta nacional de relaciones sociales del Perú 2013 y 2015 se evidenció que más del $80 \%$ de infantes y adolescentes experimentaron maltrato físico y psicológico en sus hogares y escuelas. Se observó también que más del $45 \%$ de los adolescentes sufrieron abusos sexuales (INEI, 2015).

Mediante la observación empírica, se encontró que, a nivel local, en la población Awajún, hablar de maltrato infantil no causa ninguna expectativa o preocupación, existe un profundo desconocimiento de los efectos negativos que repercute. En este sentido, consideran el uso del castigo, una forma de disciplinar y educar a los niños y niñas para que sean personas de bien. Así mismo, el abuso sexual se alimenta de los estereotipos, donde a muchas menores los responsabilizan por el acto de violación. El reglamento interno de las comunidades parece ser muy débil, en donde al agresor se les impone pagar 2000 nuevos soles y un mes de privación de libertad. Los menosprecios, humillaciones, insultos y burlas, son del vivir del día a día que los niños tienen que lidiar con ello. El abandono familiar, negligencia y la desatención se observa a simple vista en los niños y niñas sin compañía de los padres, con hambre, falta de aseo, niños que no asisten a la escuela y que muchas veces cumplen el rol de las madres a su temprana edad. Siendo estas carencias muchas veces justificadas por las condiciones económicas donde se encuentran.

En esta región, existen diferentes culturas, cada una de ellas tiene costumbres, valores y normas muy particulares, algunas están más relacionadas e influidas por civilizaciones foráneas y conceptualizaciones sobre estilos de vida que se debe practicar, sin embargo también hay distritos como el que es parte la población de estudio, donde no tienen tanta facilidad y no permiten de algún modo el contacto e influencia de otros lugares y sus formas de convivir, por lo tanto mantienen sus propias normas, en las cuales está presente el maltrato como un recurso útil para la formación de los menores.

Sin embargo, se debe tener en cuenta que todo 
maltrato a los menores, efectuado en un contexto donde se tiene el conocimiento de sus consecuencias o en una cultura donde se considera que es normal e incluso adecuado, tiene un efecto negativo sobre ellos. Partiendo de esa situación se ha trabajado esta investigación, para contribuir en el enriquecimiento de la literatura científica poniendo en evidencia un nuevo dato con respecto a una población en particular como es la etnia Awajún, así como puede servir como antecedente para implementar políticas preventivas y para profundizar los próximos estudios en la población Awajún

\section{MATERIAL Y MÉTODO}

La investigación fue de enfoque cuantitativo, de nivel relacional; de tipo temporal, fue de corte transversal y de orientación explicativa (Hernández., et al, 2018).

La población muestral estuvo constituida por los adolescentes Awajún de quinto grado de secundaria del colegio José Kistug Pujupat del distrito de Imaza, región Amazonas, Perú, matriculados en el semestre 2021, en un total de 43 estudiantes.

Para obtener los datos se utilizó la encuesta y el formulario.

La variable 1: "Maltrato infantil" fue medida por el cuestionario denominado "Test de Maltrato Infantil", con 36 proposiciones que identificó los tipos de maltrato infantil, con 4 formas: maltrato infantil físico, maltrato infantil emocional o psicológico, maltrato infantil de desatención o negligencia y maltrato sexual.

La variable 2: "Autoestima" fue medido por el Inventario de Autoestima original Forma Escolar Coopersmith adaptado por Ibañez, Hiyo en el 2018, con 58 proposiciones a las que se respondió con Sí o No, divido en 4 subescalas: área sí mismo, área social, área hogar y área escolar.

Se usó la herramienta estadística SPSS y Excel, para analizar los datos recolectados. La prueba estadística que se empleó para comprobar la relación, fue el Coeficiente de correlación de Spearman.

\section{RESULTADOS}

\section{Tabla 1}

Tipos de maltrato en adolescentes Awajún de 5to grado de secundaria. I.E José Kistug Pujupat de Chipe Cuzu. 2021

\begin{tabular}{|c|c|c|c|c|c|c|}
\hline \multirow{2}{*}{$\begin{array}{l}\text { Tipos de } \\
\text { Maltrato }\end{array}$} & \multicolumn{6}{|c|}{ Presencia } \\
\hline & $\mathbf{S i}$ & $\%$ & No & $\%$ & Total & $\%$ \\
\hline $\begin{array}{c}\text { Maltrato } \\
\text { físico }\end{array}$ & 8 & $40 \%$ & 12 & $60 \%$ & 20 & $100 \%$ \\
\hline $\begin{array}{c}\text { Emocional } \\
\text { o } \\
\text { psicológico } \\
\text { Abandono }\end{array}$ & 11 & $55 \%$ & 9 & $45 \%$ & 20 & $100 \%$ \\
\hline $\begin{array}{c}0 \\
\text { negligencia }\end{array}$ & 19 & $95 \%$ & 1 & $5 \%$ & 20 & $100 \%$ \\
\hline $\begin{array}{c}\text { Maltrato } \\
\text { sexual }\end{array}$ & 11 & $55 \%$ & 9 & $45 \%$ & 20 & $100 \%$ \\
\hline
\end{tabular}

La tabla 1 muestra que de 20 adolescentes Awajún de 5to grado de la I.E José Kistug Pujupat, el 40\% (8) han sufrido de maltrato físico, el 55\% (11) han sido víctimas de maltrato psicológico, el 95\% (19) han pasado por abandono y el 55\% (11) han sido víctimas de maltrato sexual.

\section{Tabla 2}

Niveles de autoestima en adolescentes Awajún de 5to grado de secundaria. I.E José Kistug Pujupat de Chipe Cuzu. 2021.

\begin{tabular}{cccc}
\hline Nivel de autoestima & $\mathbf{f i}$ & $\mathbf{\%}$ & $\mathbf{H i} \%$ \\
\hline Bajo & 0 & 0.0 & 0.0 \\
Promedio & 17 & 85.0 & 100.0 \\
Alto & 3 & 15.0 & 15.0 \\
\hline Total & $\mathbf{2 0}$ & $\mathbf{1 0 0 . 0}$ & \\
\hline
\end{tabular}

En la tabla 2 se observa que de 20 adolescentes Awajún de 5to grado de la I.E José Kistug Pujupat, el $85 \%$ (17) poseen una autoestima promedio, el $15 \%$ (3) tienen una autoestima alta, mientras que ningún adolescente presenta autoestima baja. 
Tabla 3

Correlación entre maltrato infantil y autoestima de adolescentes Awajún de 5to grado de secundaria. I.E José Kistug Pujupat de Chipe Cuzu. 2021

\begin{tabular}{|c|c|c|c|c|}
\hline \multicolumn{5}{|c|}{ Correlaciones } \\
\hline & & & $\begin{array}{l}\text { Maltrato } \\
\text { Infantil }\end{array}$ & Autoestima \\
\hline \multirow[t]{6}{*}{$\begin{array}{l}\text { Rho de } \\
\text { Spearman }\end{array}$} & $\begin{array}{l}\text { Maltrato } \\
\text { Infantil }\end{array}$ & $\begin{array}{l}\text { Coeficiente } \\
\text { de } \\
\text { correlación }\end{array}$ & 1.000 &,$- 522^{*}$ \\
\hline & & $\begin{array}{l}\text { Sig. } \\
\text { (bilateral) }\end{array}$ & & 0.018 \\
\hline & & $\mathrm{N}$ & 20 & 20 \\
\hline & Autoestima & $\begin{array}{l}\text { Coeficiente } \\
\text { de } \\
\text { correlación }\end{array}$ &,$- 522^{*}$ & 1.000 \\
\hline & & $\begin{array}{l}\text { Sig. } \\
\text { (bilateral) }\end{array}$ & 0.018 & \\
\hline & & $\mathrm{N}$ & 20 & 20 \\
\hline
\end{tabular}

Visualizando la tabla 3, el coeficiente de correlación de Spearman, presentó un valor menor que 0.05 $(p=0.018<0.05)$, lo que significa que se rechaza la $\mathrm{H} 0$, concluyendo que existe relación significativa al $5 \%$, entre el maltrato infantil y autoestima, el valor del coeficiente de correlación, presentó un signo negativo $\left(r_{s}=\mathbf{0} 0.522\right)$, indicando que existe una dirección inversamente proporcional entre ambas variables y cuya fuerza de asociación es moderada negativa.

\section{DISCUSIÓN}

Un $95 \%$ de la población en este estudio presentaron maltrato por Abandono o Negligencia, quiere decir que los adolescentes carecen de acompañamiento de sus padres en cuanto a la vestimenta, salud, educación, alimentación, etc. El 55\% sufrieron de maltrato sexual y emocional o psicológico, significa que los adolescentes Awajún viven bajo amenazas, humillaciones, burlas e insultos. Así mismo, viven su sexualidad de forma prematura, sin su consentimiento. Finalmente, el $40 \%$ presentaron maltrato físico, quiere decir que los adolescentes sufren de uso de fuerza excesiva e intencional causándoles lesiones.

Trigoso Gaslac. (2017). Perú. En su estudio donde determinó los tipos de maltrato que vivenciaron los estudiantes de una institución primaria, en Chachapoyas, Perú, encontró como resultado que el maltrato físico lo experimenta casi exactamente la mitad de su población, por lo tanto, se considera cómo más prevalente, en continuidad está el maltrato psicológico y de abandono y en último lugar como menos frecuente el maltrato sexual.

Fernández et al (2020). Venezuela. Realizó una investigación para encontrar las consecuencias que acarrea el maltrato infantil en un hospital de Maracaibo. En los resultados de este estudio nos muestra que el $34.54 \%$ de niños sufrían maltrato físico, el 30\% psicológico, el $26.36 \%$ representa a la ausencia y un $9.09 \%$ sexual.

Tran et al. (2016). Vietnam. En su investigación titulado: "Maltrato infantil en Vietnam: prevalencia y comparación intercultural". En los resultaron informaron que el abuso emocional fue más prevalente con un $31.8 \%$, seguido por la negligencia $25 \%$, el abuso físico $19.1 \%$, la presencia de conflictos entre los padres $15.3 \%$ y el abuso sexual $2.6 \%$. En comparación con los Países Bajos, las tasas de prevalencia de la mayoría de los tipos de maltrato infantil fueron más altas en Vietnam: la mayor diferencia fue con el abuso emocional, seguido por la negligencia y el abuso físico.

Los resultados de la presente investigación sobre la variable maltrato, indica que el tipo de maltrato por negligencia o abandono es más frecuente en la población Awajún, sin embargo, comparando con otras investigaciones nacionales e internacionales, podemos apreciar que no existe similitud de resultados, porque en el estudio de Trigoso Gaslac, el maltrato más frecuente es el físico, al igual que en el de Fernández et al., mientras que en la investigación de Tran et al. Prevalece el abuso emocional. Este índice elevado de presencia de negligencia particular en la zona, se debe a los escasos recursos económicos con los que cuentan, por lo tanto, a pesar de querer brindar mejores cuidados a sus menores no pueden hacerlo, además, su cultura no considera importante la educación y el apoyo emocional.

Del $100 \%$ del total de los adolescentes Awajún el $85 \%$ tienen un nivel de autoestima promedio, quiere decir que mantienen una actitud positiva hacia sí mismo, confían en sus capacidades a la vez que son conscientes de sus limitaciones, sin embargo, ante situaciones difíciles es deficiente su capacidad de afrontamiento, por lo tanto, le cuesta sobresalir. El $15 \%$ tienen un nivel de autoestima de nivel alto, lo que quiere decir que creen firmemente en sus capacidades y se sienten muy seguros de sí mismos, se consideran valiosos, únicos, disfrutan las actividades que realizan y un $0 \%$ presentó un nivel de autoestima bajo.

Solano. (2019). Perú. En su investigación para conocer los niveles de autoestima de los adolescentes, obtuvo un nivel de autoestima alto con $73.3 \%$, un porcentaje moderado fue con un nivel de autoestima medio con $25.7 \%$, finalmente, autoestima baja con $1 \%$.

Coyla. (2017). Perú. En su estudio para diagnosticar niveles de autoestima en los estudiantes de secundaria residentes en la Isla en Juliaca. Informó que los estudiantes presentan en su mayoría un nivel de autoestima medio con $75.8 \%$ y en menor proporción baja autoestima con $12.6 \%$. Ninguno presentó una alta autoestima.

Respecto a la autoestima, el resultado fue ampliamente predominante en nivel de autoestima 
promedio. Este resultado no coincide con los encontrados por Solano (2019) en lo cual encontró un nivel de autoestima alta, en un porcentaje muy elevado. Sin embargo, guarda relación en los niveles bajos de autoestima donde los porcentajes son casi inexistentes. Por otro lado, los resultados son similares con lo hallado por Coyla (2017), quien encontró un alto porcentaje de autoestima promedio en los adolescentes. Las similitudes y diferencias en los resultados no son discutibles debido a que los antecedentes son de una sola variable, ello implica múltiples factores que pudieron determinar los niveles autoestima.

En cuanto a la relación hallada en otros estudios, podemos encontrar que Mwakanyamale y Yizhen. (2019). Tanzania. Examinaron la relación entre el maltrato psicológico infantil y la autoestima durante la adolescencia. Demostraron que el $76.6 \%$ sufrieron maltrato psicológico, así mismo, se obtuvo una fuerte correlación positiva entre el maltrato psicológico y la autoestima. Como conclusión: los resultados evidencian que el maltrato psicológico infantil mantiene una alta prevalencia causando una baja autoestima durante la adolescencia.

Por otra parte, Quilli y Paricahua. (2019). Perú. Realizaron una investigación con estudiantes del quinto grado de primaria del colegio Divino Niño Jesús en Arequipa. Demostraron que hay una relación inversa entre el maltrato infantil y la autoestima. Por ello, quienes sufren de maltrato presentan niveles bajos de autoestima con ello queda demostrado, que mientras mayor sea el maltrato infantil menor será el nivel autoestima.

Comparando con los resultados ya encontrados, en este estudio entre las variables maltrato infantil y autoestima existe una relación significativa, con una fuerza de asociación negativa moderada, demostrando que la existencia de maltrato infantil afecta negativamente en los niveles de autoestima de los adolescentes Awajún. Corroborado por estudios realizados no solo en el Perú sino en otros países.

Mientras que Rios Ávila. (2019). Quién también realizó su investigación en Perú. En su estudio obtuvo en maltrato emocional niveles muy altos, en maltrato físico un nivel alto, en maltrato por negligencia un nivel promedio y el nivel de autoestima encontrado fue bajo. Concluyendo que no hay una relación significativa entre el maltrato infantil y autoestima.

En comparación con esta investigación, se ha demostrado lo contrario, ya que los niveles hallados oscilan entre un $95 \%$ de predominancia de abandono y una autoestima promedio, señalando de esta manera que el maltrato y autoestima van a variar de acuerdo al lugar en el que se dé y la condición cultural.

Así mismo, durante la investigación, se encontraron dos limitantes, los cuales son el de tener una población reducida, debido a que no toda la población pudo ser participe, ya que los estudiantes no estuvieron con predisposición para realizar los test y los instrumentos de medición utilizados no estaban adaptados al idioma Awajún, por lo tanto, solo los estudiantes del último año de secundaria podían entender el idioma español con mayor claridad y responder a los test.

\section{CONCLUSIONES}

Se encontró mediante el coeficiente de correlación de Spearman $(p<0.05)$ que existe relación significativa entre maltrato infantil y autoestima, con fuerza de asociación moderada negativa.

El 40\% de adolescentes Awajún de 5to grado de la I.E José Kistug Pujupat, han sufrido de maltrato físico, el $55 \%$ han sido víctimas de maltrato psicológico, el $95 \%$ han pasado por abandono y el $55 \%$ han sido víctimas de maltrato sexual.

Respecto a los niveles de autoestima, el 85\% de adolescentes Awajún poseen autoestima promedio, el $15 \%$ tienen autoestima alta, mientras que ninguno presenta autoestima baja.

\section{REFERENCIAS BIBLIOGRÁFICAS}

Amores-Villalba, A., y Mateos-Mateos, R. (2017). Revisión de la neuropsicología del maltrato infantil: la neurobiología y el perfil neuropsicológico de las víctimas de abusos en la infancia. Elseiver, 23, 8188.https://www.sciencedirect.com/scienc e/article/pii/S1135755X17300234

Arango, M., y Panesso, K. (2017). La autoestima, Proceso Humano. Psyconex, 9(14), 1-9.

Coyla, Y. (2017). Niveles de autoestima en los adolecentes de la I.E.S. José Carlos Mariategui - Isla-Juliaca. [Tesis de Grado], 54.

Defensoría del pueblo. (2019). Adjuntía para la Niñez y Adolescencia 2019. https://www.defen soria.gob.pe/ wpcontent/uploads/2020/01/reportemami s-2019.pdf

Fernández, G., Farina, P., Arráiz, C., y Troya, E. (2020). Consecuencias del maltrato infantil en un hospital de Maracaibo Venezuela. Revista de ciencias sociales, XXVI(1). https://www.redalyc.org/ journal/280/28063104016/html/ 
Hernández, R., y Mendoza, C. (2018). Metodología de la investigación. Las rutas cuantitativa, cualitativa y mixta. Mexico: $M$ c G R A W - H I L L. h t t p : // repositorio.uas b.edu. bo:8080/bitstream/54000/1292/1/Hernán dez-Metodología de la investigación.pdf

Hiyo, C. (2018). Propiedades psicométricas y estandarización del inventario de autoestima de Coopersmith en escolares de secundaria de lima sur: Chorrillos, Surco y Barranco [UNIVERSIDAD A U T Ó N O M A D E L P E R Ú ] . http://repositorio.autonoma.edu.pe/handl e/AUTONOMA/557

Instituto Nacional de Estadística e Informática (2015) Encuesta Nacional sobre Relaciones Sociales -ENARES 2013 y 2015. https://www.inei.gob.pe/media/ MenuRecursivo/publicaciones_digitales/ Est/Lib1390/libro.pdf

Mwakanyamale, A. A., \& Yizhen, Y. (2019). Psychological maltreatment and its relationship with self-esteem and psychological stress among adolescents in Tanzania: a community based, crosssectional study. BMC psychiatry, 19(176). https://doi.org/10.1186/s12888-0192139-y

Organización Mundial de la Salud. (2020). Maltrato infantil. https://www.who.int/es/newsroom/fact-sheets/detail/child-maltrea tment

Organización Panamericana de Salud .(2016). Siete estrategias para poner fin a la violencia contra los niños y las niñas. Washigton: OPS. https://iris.paho.org/bitstream /handle/10665.2/33741/9789275319413spa.pdf? sequence $=7 \&$ isAllowed $=\mathrm{y}$

Pérez, H. (Febrero-Julio de 2019). Autoestima, teorías y su relación con el éxito. Alternativas Psicología(41), 22-31. https:/www.alternativas.me/attachments /article/190/2.\%20Autoestima,\%20teor\% C3\%ADas\%20y\%20su\%20relaci\%C3\% B3n $\% 20$ con $\% 20$ el $\% 20 \%$ C3\%A9xito $\% 2$ 0personal.pdf

Quilli, M. R., y Paricahua, J. V. (2019). Correlación entre el maltrato infantil y la autoestima de los estudiantes del quinto grado de educación primaria de la institución educativa particular "Divino niño Jesús" del distrito de Chala, 2018. [Tesis de pregrado]. Universidad Nacional de San Agustín de Arequipa, Perú. http://repositorio.unsa.edu.pe/bitstream/h andle/UNSA/10699/EDCapqumr1.pdf?se quence $=3 \&$ is Allowed $=\mathrm{y}$

Rios Avila, B. M. (2019). Maltrato infantil y su relación con la autoestima en escolares de la Institución educativa primaria $N^{\circ}$ 53004 Miguel Grau - Iberia, 2018. Universidad nacional del Altiplano, Perú . http://repositorio.unap.edu.pe/handle/UN AP/13995

Solano Ilizarbe, Y. J. (2019). Nivel de autoestima en estudiantes adolescentes pertenecientes al distrito de Pampas - Huancavelica. Universidad San Ignacio de Loyola, 34. http://repositorio.usil.edu.pe/handle/USI L/9423

Tran NK, Alink LRA, Van Berkel SR, Van IJzendoorn MH. Maltrato infantil en Vietnam: prevalencia y comparación intercultural. Revista de agresión, maltrato y trauma. 2016; 26 (3): 211-30. https:// doi.org/10.1080/10926771.201 6.1250851

Trigoso Gaslac, L. M. (2017 ). Tipos de maltrato infantil que presentan los estudiantes de la institución educativa primaria Pedro Castro Alva $N^{\circ}$ 18006, Chachapoyas 2017. Tesis de pregrado. Universidad Nacional Toribio Rodríguez de Mendoza, Chachapoyas, Perú. http://repositorio. untrm.edu.pe/bitstream/handle/UNTRM/ 1245/INFORME\%20FINAL.pdf?sequen ce $=1 \&$ is Allowed $=y$ 